\section{Wing origami}

Origami-based design techniques have applications ranging from nanoscale devices to architectural structures. But conventional techniques have a limited range of folding patterns. Writing in Science, Faber et al. report an approach that overcomes this restriction (J. A. Faber et al. Science 359, 1386-1391; 2018).

The authors were inspired by the amazing folding abilities of earwig wings (pictured). They discovered that protein-rich joints in the wings behave like springs, and mimicked this design in synthetic structures using mechanical springs. Such structures show folding patterns and functionalities that were impossible using previously available methods. Ryan Wilkinson

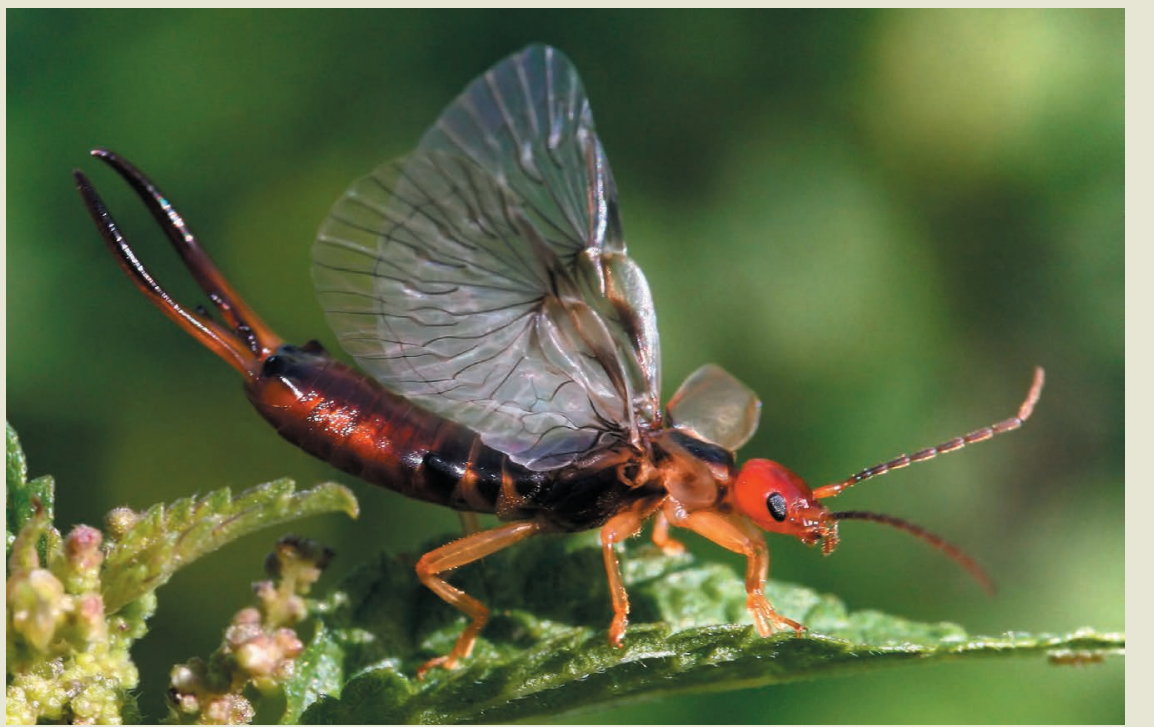

IMMUNOLOGY

\title{
Killer T cells show their kinder side
}

The immune system protects the body by responding to invading organisms. But how is an attack on useful resident microbes prevented? A pathway has now been identified that allows immune cells to sense and respond to beneficial bacteria.

\section{PAUL KLENERMAN \& GRAHAM OGG}

$\mathrm{T}$ he immune system performs a difficult balancing act. It must respond rapidly to dangerous microorganisms that threaten the host, yet also coexist peacefully with the large array of microorganisms that colonize barrier surfaces such as those of the skin and gut. Writing in Cell, Linehan et al. ${ }^{1}$ examine the interactions between immune cells and a bacterium that normally resides on the skin, and identify a signalling pathway that governs the immune response to such microbes.

Most immunological research has focused on how immune cells recognize and eliminate viral and bacterial pathogens, and the insights obtained have led to the development of many immune-based therapies. However, attention has also turned to microbial communities at barrier surfaces and the long-term influence they exert on host immune responses. Accordingly, Linehan and colleagues studied the skin-dwelling bacterium Staphylococcus epidermidis in mice and rhesus monkeys, as well as analysing human-tissue samples from the clinic.

Staphylococcus epidermidis belongs to a group of microorganisms termed commensals, and usually colonizes human skin without causing disease. However, in premature newborns and people who are in an immunosuppressed state or who have received artificial joints, S. epidermidis can cause severe disease or chronic infection ${ }^{2}$. Furthermore, even though the bacterium is normally harmless, it is not ignored by the immune system. In mouse skin, the response ${ }^{3}$ to an initial encounter with certain strains of $S$. epidermidis is dominated by a type of immune cell called a cytotoxic $\mathrm{CD}^{+} \mathrm{T}$ cell, also known as a killer $\mathrm{T}$ cell. Linehan and colleagues asked two key questions about this response, and the answers they obtained are illuminating.

The first question was, how do T cells recognize S. epidermidis? A response to bacteria is thought to occur when a $\mathrm{T}$ cell recognizes a bacterial peptide fragment bound to the 'classical' class Ia protein component of the major histocompatibility complex (MHC), which presents such fragments to immune cells. However, using a mouse model system, Linehan and colleagues found that a 'nonclassical' protein, H2-M3, which belongs to the MHC class Ib family, was instead involved in $\mathrm{MHC}$ presentation in the response to S. epidermidis. $\mathrm{H} 2-\mathrm{M} 3$ can present ${ }^{4}$ bacterial peptides in which the amino-acid residue methionine has been modified by addition of an $N$-formyl group.

The other question Linehan and colleagues sought to answer was whether this nonclassical immune-recognition pathway might also be coupled to 'unconventional' T-cell behaviour. The team investigated this by comparing gene-expression patterns of $\mathrm{CD}^{+} \mathrm{T}$ cells responding to $S$. epidermidis with those of other types of T-cell population in the mouse skin that did not respond to the bacterium. The authors found that the responsive $\mathrm{CD}^{+} \mathrm{T}$ cells expressed genes associated with wound healing and tissue repair (Fig. 1). This was surprising because responding $\mathrm{CD} 8^{+}$ T cells would be expected to express genes associated with activation of the pathways involved in killing unwanted cells or in the release of pro-inflammatory signalling molecules.

Using an in vivo mouse model of skinwound healing in association with S. epidermidis colonization, the authors demonstrated that the tissue-repair gene-expression signature in responding $\mathrm{T}$ cells was linked to promotion of a healing response. Thus, immune recognition of bacterial residents of the skin can induce a form of immunity that does not directly retaliate against the bacterium, but instead aids tissue repair.

The authors' finding adds to a growing recognition of the diverse functions of other unconventional $\mathrm{T}$ cells that are found at barrier sites and that can recognize evolutionarily conserved microbial molecules. These cells include, for example, mucosal-associated invariant $\mathrm{T}$ cells, which are abundant in humans and induced by commensals, and which share several features ${ }^{5}$ of the T-cell population studied by Linehan and colleagues - such as the production of the signalling molecule interleukin-17A. 